\title{
The Analysis of Alfvén Wave Current Drive and Plasma Heating in TCABR Tokamak
}

\author{
L.F. Ruchko, E.A. Lerche, R.M.O. Galvão, A.G. Elfimov, I.C.Nascimento, \\ W.P. de Sá, E. Sanada, J.I. Elizondo, A.A. Ferreira, E.A. Saettone, \\ J.H.F. Severo, V. Bellintani, and O.N. Usuriaga \\ Instituto de Física, Universidade de São Paulo, \\ Caixa Postal 66318, CEP 05315-970, São Paulo, Brasil
}

Received on 26 June, 2001

\begin{abstract}
The results of experiments on Alfvén wave current drive and plasma heating in the TCABR tokamak are analyzed with the help of a numerical code for simulation of the diffusion of the toroidal electric field. It permits to find radial distributions of plasma current density and conductivity, which match the experimentally measured total plasma current and loop voltage changes, and thus to study the performance of the RF system during Alfvén wave plasma heating and current drive experiments. Regimes with efficient RF power input in TCABR have been analyzed and revealed the possibility of noninductive current generation with magnitudes up to $\sim 8 \mathrm{kA}$. The increase of plasma energy content due to RF power input is consistent with the diamagnetic measurements.
\end{abstract}

\section{Introduction}

The knowledge of the magnitude and radial position of noninductively driven toroidal current in a tokamak plasma, which is generated by Alfvén waves, is of great importance both for optimization of the current drive scheme itself and for tokamak operation. The most commonly used method for determination of the magnitude of the noninductively driven current consists of the analysis of the loop voltage response to the application of a noninductive driving force. If the total current is maintained constant and a stationary state is obtained, then the magnitude of the driven current can be easily calculated through the loop voltage drop at the plasma boundary. More complicated calculations are necessary in the case when stationary conditions are not achieved.

The problem of toroidal current diffusion in the presence of external non-Ohmic current drive was analyzed in the review [1]. It was pointed out there that characteristic time scale of plasma current build-up, which is connected with transformation of the energy of external noninductive current source into the energy of the poloidal magnetic field of the plasma column, can be divided in two parts, $\tau_{\text {ext }}$ and $\tau_{i n t}, \tau_{\text {int }}<\tau_{\text {ext }}$, in accordance with the division of the total poloidal magnetic field energy $W_{t}$ in two parts $W_{t}=W_{i n t}+W_{\text {ext }}$, where $W_{\text {int }}$ is the energy of the magnetic field inside plasma and $W_{e x t}$ is the energy of the magnetic field outside the plasma column. Usually $W_{\text {int }}$ amounts to $25-30 \%$ of $W_{t}$. The characteristic time scale can be defined as the ratio of the magnetic field energy, $W_{t}=\frac{L I^{2}}{2}$, to ohmic power, $R I^{2}$, which is dissipated in plasma due to the flow of the electric current that creates this magnetic field. This time corresponds to the $\frac{L}{R}$ time of an electrical scheme with resistor $R$ and inductor $L$ connected in series. The smaller time scale $\tau_{\text {int }}$ corresponds to the time that is necessary for termination of all transient processes inside plasma column, that is during this time the rebuilding of the profile of the plasma current is completed and the subsequent changes of total plasma current $I$ are accomplished with conservation of the current profile $j(r)$. The larger time scale $\tau_{e x t}$ corresponds to the time which is necessary for the total current to reach a stationary value. In the framework of a $0-D$ numerical model, in which the plasma is represented as resistance and inductance connected in series, only the processes with characteristic time scales $\tau \geq \tau_{\text {ext }}$ can be analyzed. The process of toroidal current redistribution with a time scale $\tau \leq \tau_{\text {int }}$ has to be analyzed by solving an 1-D plasma transport model.

Current diffusion and toroidal electric field response to non-Ohmic current drive in the framework of a simplified 1-D model was studied in [2]. In this paper, a model consisting of a cylindrical plasma column in an uniform axial magnetic field was considered, the plasma quantities and electric field were assumed to be both axially and azimuthally symmetric and, for simplifica- 
tion of numerical calculations, the plasma conductivity was taken as known, rather than self-consistently found from the complete set of transport equations. In order to include the effects that depend on conductivity profiles, analytical solutions were complemented by numerically found eigenfunctions of diffusion boundary problem. These results were used for interpretation of experimental data on Alfvén wave current drive in the PHAEDRUS-T tokamak [3] and have demonstrated a correct description of the evolution of the electric field. They have shown also that the time behavior of loop voltage response to application of non-Ohmic current drive can be used to find out the radial position of the noninductively-driven current.

In this paper we present a numerical code that has been developed specially for analysis of the experimental data on noninductive-current drive by Alfvén waves in the TCABR tokamak [4]. We use a 1-D plasma model similar to [2]. This completely numerical procedure for solution the diffusion evolution for the electric field has no computational limitations on plasma profiles and temporal behavior of the driving force. It permits to find radial distributions of plasma current density and conductivity, which match the experimentally measured total plasma current and loop voltage changes, and thus to study the performance of the RF system during Alfvén wave plasma heating and current drive experiments. Different regimes with efficient RF power input in TCABR have been analyzed and revealed the possibility of noninductive current generation with magnitudes up to $\sim 8 \mathrm{kA}$.

\section{Numerical model}

We use the usual power balance assumption that all plasma parameters depend on time and on one spatial coordinate, which determines the circular magnetic surfaces. That is, in the following all local quantities such as plasma temperature, current density, input power etc., correspond to averaged values on magnetic surfaces. Further simplifications on the numerical calculations are made assuming that the temperature and conductivity do not come self-consistently from power balance equations, but can be modified to fit experimental data or can be predefined functions of time and radius $T(r, t)$ and $\sigma(r, t)$.

The plasma current response to noninductive cur- rent drive is governed by Maxwell equations

$$
\operatorname{rot} \vec{H}=\frac{4 \pi}{c} \sigma \vec{E}+\frac{4 \pi}{c} \vec{j}_{d}
$$

and

$$
\operatorname{rot} \vec{E}=-\frac{1}{c} \frac{\partial \vec{H}}{\partial t}
$$

Here we assume that the toroidal component of Ohm's law has the form

$$
j=j_{d}+\sigma(r, t) E
$$

where $j, j_{d}$ and $E$ are axial total current density, noninductively driven current density, and electric field, respectively. From these equations, we obtain the diffusion equation for the electric field:

$$
\Delta E=\frac{4 \pi}{c^{2}}\left(\frac{\partial \sigma}{\partial t} E+\sigma \frac{\partial E}{\partial t}+\frac{\partial j_{d}}{\partial t}\right)
$$

After the substitutions $r=x a, \frac{c^{2}}{4 \pi \sigma a^{2}}=k$, and $j_{d}=\sigma S$, the following inhomogeneous diffusion equation follows

$$
\begin{gathered}
\frac{\partial E}{\partial t}=k(x, t) \frac{1}{x} \frac{\partial}{\partial x}\left(x \frac{\partial E}{\partial x}\right)-\frac{1}{\sigma(x, t)} \frac{\partial \sigma(x, t)}{\partial t} E \\
-\frac{\partial S}{\partial t}-\frac{1}{\sigma(x, t)} \frac{\partial \sigma(x, t)}{\partial t} S
\end{gathered}
$$

Here we introduced the "effective" field $S(x, t)$, which corresponds to noninductive current drive force and is determined by the input RF power and current drive efficiency.

This equation has to be solved with boundary conditions

$$
\left.\frac{\partial E}{\partial x}\right|_{x=0}=0,\left.\quad \frac{\partial E}{\partial x}\right|_{x=1}=\frac{2}{c^{2}} \frac{\partial I_{p}}{\partial t}
$$

and initial condition $E(x, 0)=\varphi(x)$, where $I_{p}$ is the total plasma current.

The boundary value problem Eq.5-6 is solved using the Crank-Nicolson numerical scheme, which gives high accuracy and is unconditionally stable. Some details of numerical scheme are given below.

As first step, we divide the spatial interval $0 \leq x \leq 1$ into $N-1$ elements $h$ by $N$ points $i=1,2 \ldots N$ and $h=\frac{1}{N-1}$; then $x_{i}=(i-1) / h$. In Eq.5 we discretizy space and time in increments of $h$ and $\tau$, respectively, and obtain, for the inner points $2 \leq i \leq N-1$, the following equation: 


$$
\begin{aligned}
\frac{E_{i}^{n+1}-E_{i}^{n}}{\tau} & =\frac{D_{i}}{h^{2}}\left[E_{i+1}^{n}+E_{i-1}^{n}-2 E_{i}^{n}+\frac{h\left(E_{i+1}^{n}-E_{i-1}^{n}\right)}{2 x_{i}}\right]-\frac{E_{i}^{n}}{\sigma_{i}^{n}} \frac{\sigma_{i}^{n+1}-\sigma_{i}^{n}}{\tau} \\
& -\frac{S_{i}^{n}}{\sigma_{i}^{n}} \frac{\sigma_{i}^{n+1}-\sigma_{i}^{n}}{\tau}-\frac{S_{i}^{n+1}-S_{i}^{n}}{\tau} .
\end{aligned}
$$

Here $E_{i}^{n}=E\left(x_{i}, t_{n}\right), D_{i}=k\left(x_{i}, t_{n}\right), \sigma_{i}^{n}=\sigma\left(x_{i}, t_{n}\right), S_{i}^{n}=S\left(x_{i}, t_{n}\right)$. After substitution $x_{i}=(i-1) h$, we get

$$
\begin{aligned}
E_{i}^{n+1} & =E_{i}^{n}+\frac{\tau D_{i}}{h^{2}}\left[E_{i+1}^{n}+\frac{E_{i+1}^{n}}{2(i-1)}-\left(2+\frac{h^{2}}{\tau D_{i}} \frac{\left(\sigma_{i}^{n+1}-\sigma_{i}^{n}\right)}{\sigma_{i}^{n}}\right) E_{i}^{n}+E_{i-1}^{n}-\frac{E_{i-1}^{n}}{2(i-1)}\right] \\
& -S^{n+1}+S^{n}
\end{aligned}
$$

For outer points $i=1$ and $i=N$, we use boundary conditions at $x=0 \quad\left(\left.\frac{\partial E}{\partial x}\right|_{x=0}=0\right)$ and at $x=1\left(\left.\frac{\partial E}{\partial x}\right|_{x=1}=\right.$ $\frac{2}{c^{2}} \frac{\partial J_{p}}{\partial t}$ ) and obtain the additional relations

$$
\begin{gathered}
E_{1}^{n}=E_{2}^{n} \\
E_{N}^{n}=E_{N-1}^{n}+h \frac{2}{c^{2}} \frac{J_{p}^{n+1}-J_{p}^{n+1}}{\tau} \\
E_{i}^{n+1}=E_{i}^{n}+\chi_{i} \sum_{k=i-1}^{i+1} \mathbf{Z}_{\mathbf{i k}} \mathbf{E}_{\mathbf{k}}^{\mathbf{n}}-\left(\mathbf{S}^{\mathbf{n}+\mathbf{1}}-\mathbf{S}^{\mathbf{n}}\right)
\end{gathered}
$$

Here $\chi_{i}=\frac{\tau D_{i}}{h^{2}}$ and the operator $\mathbf{Z}_{\mathbf{i k}}$ has the following tridiagonal structure

$$
\mathbf{Z}_{\mathbf{i k}}=\delta_{\mathbf{i}, \mathbf{k}-\mathbf{1}}\left(\mathbf{1}-\frac{\mathbf{1}}{\mathbf{2 ( \mathbf { i } - \mathbf { 1 } )}}\right)+\delta_{\mathbf{i}, \mathbf{k}}\left(-\mathbf{2}-\frac{\mathbf{1}}{\chi_{\mathbf{i}}} \frac{\left(\sigma_{\mathbf{i}}^{\mathbf{n}+\mathbf{1}}-\sigma_{\mathbf{i}}^{\mathbf{n}}\right)}{\sigma_{\mathbf{i}}^{\mathbf{n}}}\right)+\delta_{\mathbf{i}, \mathbf{k}+\mathbf{1}}\left(\mathbf{1}+\frac{\mathbf{1}}{\mathbf{2 ( \mathbf { i } - \mathbf { 1 } )}}\right) .
$$

The operator elements $\mathbf{Z}_{\mathbf{1}, \mathbf{k}}$ and $\mathbf{Z}_{\mathbf{N}, \mathbf{k}}$ have to be determined through the boundary conditions at $x=0$ and $x=1$ :

$$
\begin{array}{llll}
\left.\frac{\partial E}{\partial x}\right|_{x=0} & =0 & \Longrightarrow & E_{1}^{n}=E_{2}^{n} \\
\left.\frac{\partial E}{\partial x}\right|_{x=1}=\frac{2}{c^{2}} \frac{\partial J_{p}}{\partial t} & \Longrightarrow & E_{N}^{n}=E_{N-1}^{n}+h \frac{2}{c^{2}} \frac{J_{p}^{n+1}-J_{p}^{n+1}}{\tau}
\end{array}
$$

Now it is possible to define the operator $\Psi_{i, k}$ for $\quad 1 \leq i \leq N$ :

$$
E_{i}^{n+1}=\sum_{k=1}^{N}\left[\Psi_{i, k} E_{k}^{n}+\delta_{i, k} \delta_{k, N} h \frac{2\left(J_{p}^{n+1}-J_{p}^{n}\right)}{\tau}\right]-\left(S_{k}^{n+1}-S_{k}^{n}\right)
$$

where

$$
\Psi_{i, k}=\delta_{i, k+1} \chi_{i}\left(1-\frac{1}{2(k-1)}\right)+\delta_{i, k}\left(1-2 \chi_{i}-\frac{\left(\sigma_{i}^{n+1}-\sigma_{i}^{n}\right)}{\sigma_{i}^{n}}\right)+\delta_{i, k-1} \chi_{i}\left(1+\frac{1}{2(k-1)}\right)
$$

Eq.14 represents explicit FTCS numerical scheme for the given partial differential equation (PDE). In the next step we separate time and spatial dependencies and express Eq.14 in the form

$$
E_{i}^{n+1}=E_{i}^{n}+\sum_{k} H_{i, k} E_{k}^{n}+R_{i}^{n+1}-R_{i}^{n}
$$

where $R_{i}^{n}=\delta_{i, N} \frac{h}{c^{2}} \frac{2 J_{p}^{n}}{\tau}-S_{i}^{n}$ is a known matrix, which is determined by the driving current source and by total current evolution, and

$$
H_{i, k}=\left(1-\delta_{i, 1}\right)\left(1-\delta_{i, N}\right) \Psi_{i, k}+\delta_{i, k} \delta_{k, 1} \Psi_{i+1, k}+\delta_{i, k} \delta_{k, N} \Psi_{i-1, k}-\delta_{i, k}
$$


It is useful to express Eq.16 in vector form

$$
\mathbf{E}^{\mathbf{n}+\mathbf{1}}=(\mathbf{I}+\mathbf{H}) * \mathbf{E}^{\mathbf{n}}+\mathbf{R}^{\mathbf{n}+\mathbf{1}}-\mathbf{R}^{\mathbf{n}}
$$

where $\mathbf{I}$ is the unitary operator. $\mathbf{E}^{\mathbf{n}+1}$

Now we apply the operator $\mathbf{H}$ to the future value

$$
\mathbf{E}^{\mathbf{n}+\mathbf{1}}=\mathbf{E}^{\mathbf{n}}+\mathbf{H} * \mathbf{E}^{\mathbf{n}+\mathbf{1}}+\mathbf{R}^{\mathbf{n}+\mathbf{1}}-\mathbf{R}^{\mathbf{n}}
$$

and, solving for $\mathbf{E}^{\mathbf{n}+\mathbf{1}}$, we obtain implicit FTCS scheme for the given partial differential equation

$$
\mathbf{E}^{\mathbf{n}+\mathbf{1}}=(\mathbf{I}-\mathbf{H})^{-\mathbf{1}} * \mathbf{E}^{\mathbf{n}}+\mathbf{R}^{\mathbf{n}+\mathbf{1}}-\mathbf{R}^{\mathbf{n}} .
$$

After finding the average of Eq.18 and Eq.20 and solving for $\mathbf{E}^{\mathbf{n}+\mathbf{1}}$, we finally get the Crank-Nicolson scheme for solving our partial differential problem

$$
\mathbf{E}^{\mathbf{n}+\mathbf{1}}=\left(\mathbf{I}-\frac{\mathbf{1}}{\mathbf{2}} \mathbf{H}\right)^{-\mathbf{1}}\left[\left(\mathbf{I}+\frac{\mathbf{1}}{\mathbf{2}} \mathbf{H}\right) \mathbf{E}^{\mathbf{n}}+\mathbf{R}^{\mathbf{n}+\mathbf{1}}-\mathbf{R}^{\mathbf{n}}\right]
$$

This scheme was used in the numerical simulations of the electric field diffusion. The numerical procedure included reading the experimental data on the measured plasma current, which were stored by the data acquisition system of TCABR. Then, after differentiation, these data were used as boundary conditions for the numerical code(Eq.6). After the numerical solution of equation Eq.21, we obtain the evolution of radial profile of the plasma current density and of the longitudinal electric field during the discharge. Both ohmic heating and RF heating and current drive phases can be analyzed. The numerical analysis has been performed in the following order.

1. Reading experimental data set for the plasma current, which was obtained during a plasma discharge in TCABR;

2. Smoothing of the experimental data and finding the derivative of the plasma current;

3. Numerical solution of partial differential equations for electric field diffusion with obtained boundary conditions and finding the time evolution of calculated loop voltage;

4. Analysis of simulation results and, if necessary, repetition of calculations with modified plasma parameters, which include electron temperature profile and noninductive driving force, until reasonable fitting to experimentally measured loop voltage data is obtained.

As it was shown in [2],[3] the analysis of loop voltage response to noninductive plasma current drive permits to find both the amplitude of the driven current and its radial localization.

\section{The analysis of plasma dis- charge with Alfvén wave heating and current drive}

\section{III.1 Experimental setup}

The basic regimes for Alfvén wave plasma heating and current drive experiments had following parameters: toroidal magnetic field $B_{0}=1.1 \mathrm{~T}$ on axis, major plasma radius $R=0.61 \mathrm{~m}$, minor radius $a=0.18 \mathrm{~m}$, plasma current $I_{P}=70$ to $95 \mathrm{kA}$, edge safety factor $q(a) \simeq 3.5$ to 5.5 , line averaged plasma density in the range $\left\langle n_{e}\right\rangle=(0.9$ to 3.5$) \times 10^{19} \mathrm{~m}^{-3}$, working gas hydrogen.

The used diagnostic tools include conventional electrotechnical measurements of the loop voltage $U$, plasma current $I_{P}$, plasma column shift $(d R, d Z)$, plasma energy content $\langle n T\rangle$ by diamagnetic signal and plasma equilibrium parameters, fast bolometer measurements of plasma losses, and optical measurements of visible radiation intensity. The line averaged plasma density was measured by multi-channel microwave interferometer. The ion saturation current in the limiter shadow was monitored by a movable Langmuir probe.

The complete description of the RF system of TCABR tokamak is given in [4]. In the present experiments only one module of the Alfvén wave antenna system has been used. It was powered by the 4-phase RF generator, which has nominal output power $\widetilde{P} \leq 1$ $\mathrm{MW}$ in the frequency range $f=3$ to $5.5 \mathrm{MHz}$. It provided RF pulses with duration $\tau \leq 10 \mathrm{msec}$. The scheme of antenna straps feeding is shown in Fig.1.

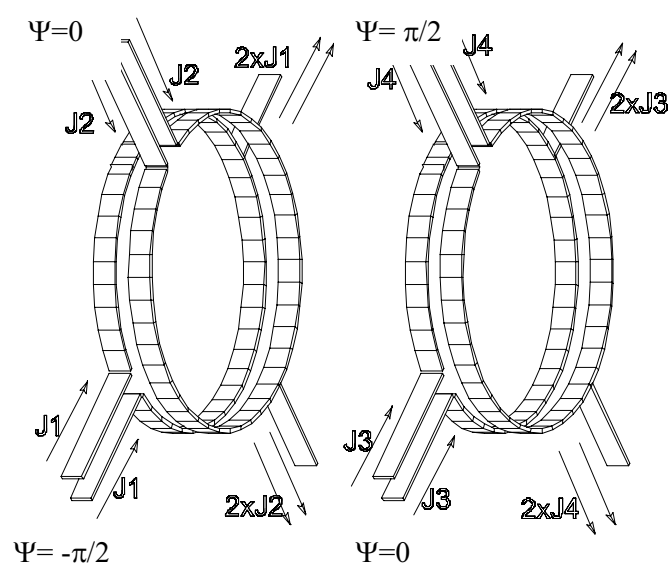

Figure 1. Schematic presentation of antenna straps feeding by RF currents. 
The spatial spectrum of the excited RF field (wave numbers $M, N$ ) and its helicity was controlled by the phase of RF feeding currents. In the course of RF experiments, different antenna configurations were tested. As it is seen in Fig.1, the antenna module of TCABR has four pairs of half loops, which can be fed independently by the RF currents. It allows to excite the Alfvén waves with different mode structure $( \pm M, \pm N)$ and to choose the desired helicity of excited wave $(M \times N>0$ or $M \times N<0)$. In the Fig. 2 there are shown schematically the direction of toroidal magnetic field $B_{T}$, plasma ohmic current $I p$ and the wave vector $\vec{k}$, which are typical for tokamak TCABR. The line determines the position of the RF wave front, which is set by phasing of the antenna feeding currents. For the present case, the helicity of the excited wave is negative $(M \times N<0)$. The antenna current feeding for this case is shown in Fig.1. Theoretical calculations predict more efficient Alfvén wave heating for this case because the projection of the wave vector on the total magnetic field $k_{/ /}$has larger value than in the case of positive helicity $(M \times N>0)$. This result appeared because of the direction of ohmic current in the TCABR tokamak, which is opposite to main toroidal magnetic field.

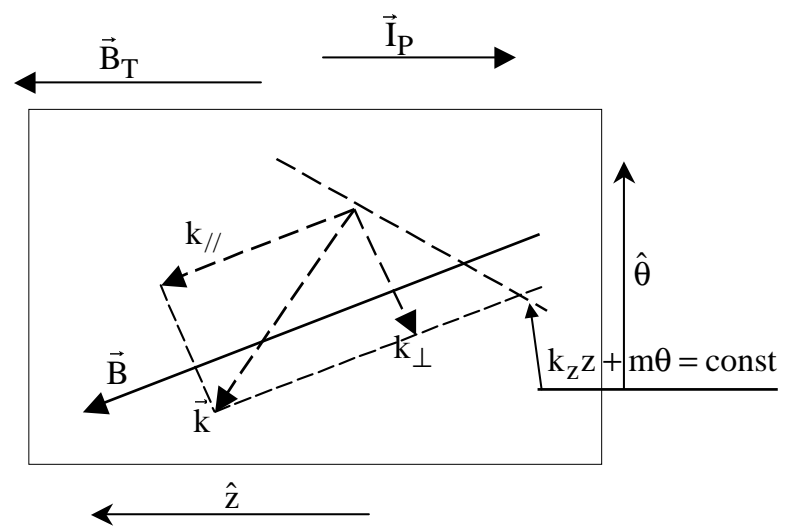

Figure 2. Schematic presentation of tokamak TCABR magnetic field structure and antenna helicity choosing.

\section{III.2 Analysis of antenna phasing influ- ence on excited wave spectrum}

The position $r_{S}$ of the zone where the RF power deposition occurs, during Alfvén wave excitation, is determined by the wave dispersion relation $\omega=k_{/ /}\left(r_{S}\right) C_{A}\left(r_{S}\right) \sqrt{\left(1-\omega^{2} / \omega_{c i}^{2}\right)}$. Here $C_{A}\left(r_{S}\right)=\frac{B}{\sqrt{4 \pi \rho\left(r_{S}\right)}}$ is the Alfvén velocity and $k_{/ /}=$ $\frac{1}{R}\left(n+\frac{m}{q\left(r_{S}\right)}\right)$ is the parallel component of wave vector. It is seen that both plasma parameters and RF system characteristics can influence $r_{S}$.

The complete antenna system of TCABR tokamak, which has to be composed of four antenna modules, ensures selective mode excitation and makes it possible to control the radial profile of the RF power deposition. This advantage of Alfvén wave heating and current drive scheme can be used for plasma confinement and stability control in the investigations that are carried out in the programs of advanced tokamak concept. The utilization of only one antenna module does not permit to excite a monochromatic spatial spectrum of Alfvén modes and to realize completely the advantages of this plasma RF heating scheme. In this case, different modes can be excited simultaneously and they can deposit $\mathrm{RF}$ power in different plasma radial regions.

a)

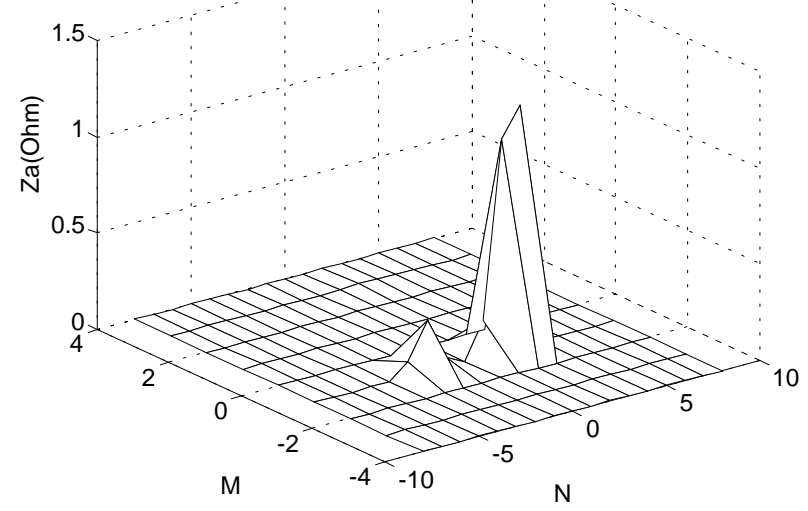

b)

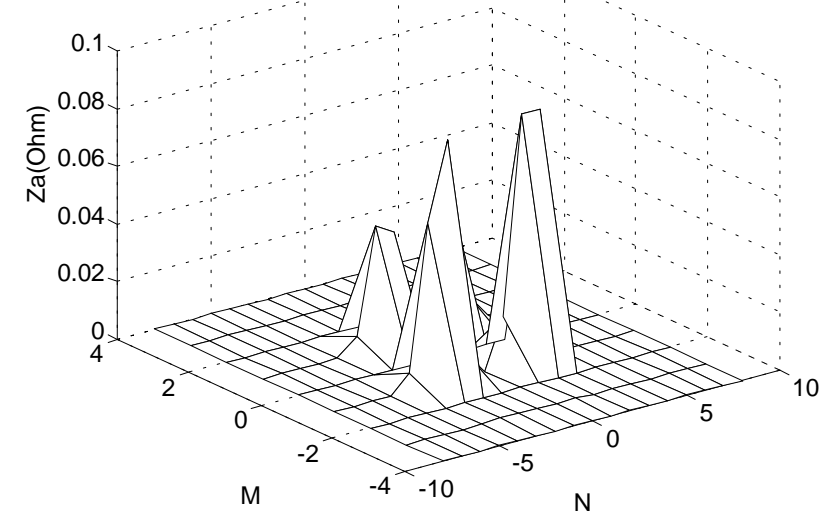

Figure 3. Wave spectrum of antenna impedance for one antenna module: a) four pairs of half-loops are powered by RF currents with $\pi / 2$ phasing, b) one pair of half-loops is powered.

The resulting radial profile of $\mathrm{RF}$ power deposition depends strongly on antenna current phasing. With correctly chosen antenna current phasing, a rather monochromatic wave spectrum can be excited. The dependence of the impedance of antenna, which is formed 
by one module with $\pi / 2$ phasing of antenna currents, on the excited mode structure is shown in Fig.3a. It is seen that the maximum RF power deposition goes through excitation of modes grouped around $M=-1, N=2,3$. They deposit their energy in determined plasma radial positions and permit to generate a noninductive current in the direction of plasma ohmic current and to change the radial profile of safety factor $q(r)$. For comparison, the impedance wave spectrum, which is excited by the antenna module when only one pair of half-loops is powered, is shown in the Fig.3b. In this case many plasma modes are excited simultaneously and it is difficult to control radial position of the power deposition zone.

The dependence of radial distribution of dissipated RF power on plasma central density for antenna module of TCABR is presented in Fig.4. The calculations have been carried out with the help of a MHD numerical code [4] [5] for one antenna module with current phasing, which is shown in Fig.1, and which corresponds to excitation of a wave with negative helicity $(M \times N<0)$. It is seen that there are regions of plasma parameters which permit efficient plasma heating with RF power deposition near the plasma column center.

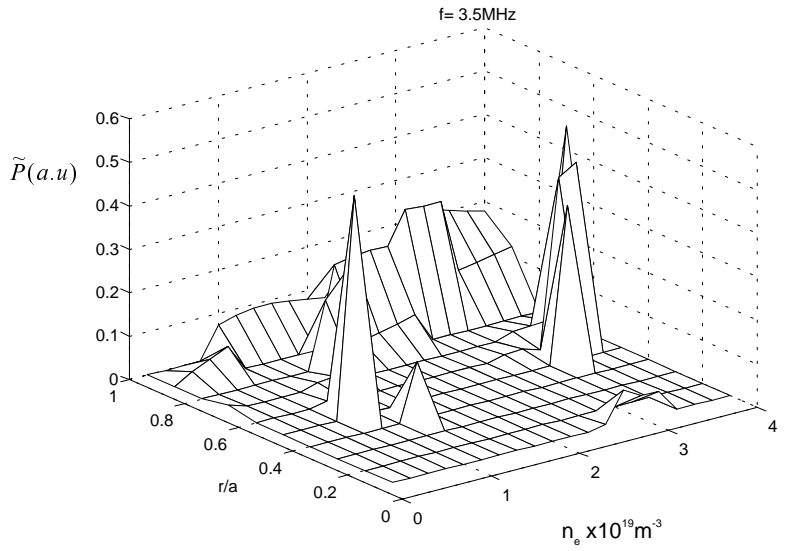

Figure 4. The radial dependence of RF power input as function of plasma central density $\mathrm{n}_{e(0)}$.

\section{III.3 Analysis of loop voltage changes during RF power input in tokamak plasma}

For the analysis, we have chosen the plasma discharge shot \#4893 in the TCABR tokamak, where efficient antenna - plasma coupling was observed. The traces of some experimental signals are shown in Fig.5. The Alfvén wave antennae were powered at time moment $t_{1}=55 \mathrm{msec}$, and the RF pulse duration was $10 \mathrm{msec}$, approximately. The details of the discharge are shown in Fig.6. It is seen that in the course of the Alfvén wave excitation some increase of total plasma current I $p$ can be observed, while the loop voltage $U$ falls down. This effect can be explained both by an electron temperature increase due to Alfvén wave heating and by noninductive current drive. In order to separate these two effects, we have to carry out the analysis of the loop voltage drop and compare the results with the independent measurements of plasma temperature evolution.

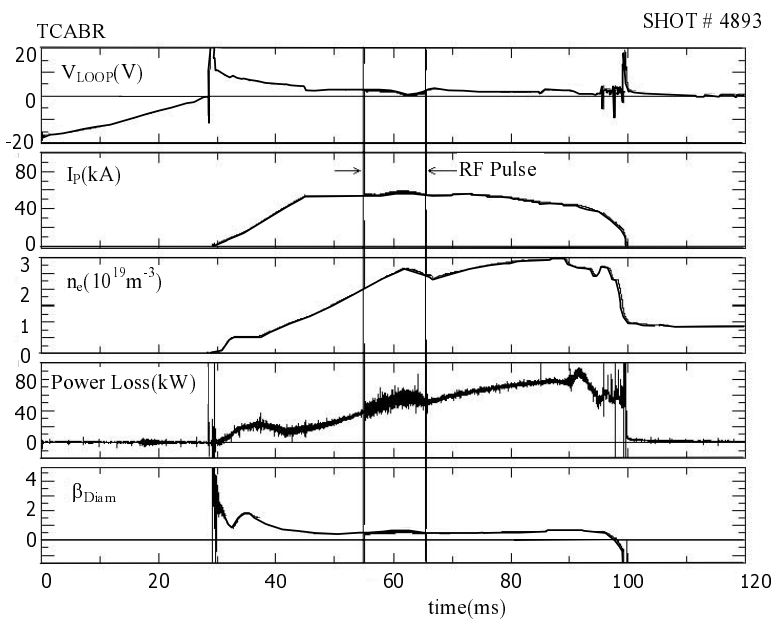

Figure 5. Plasma discharge \#4893 in TCABR tokamak.

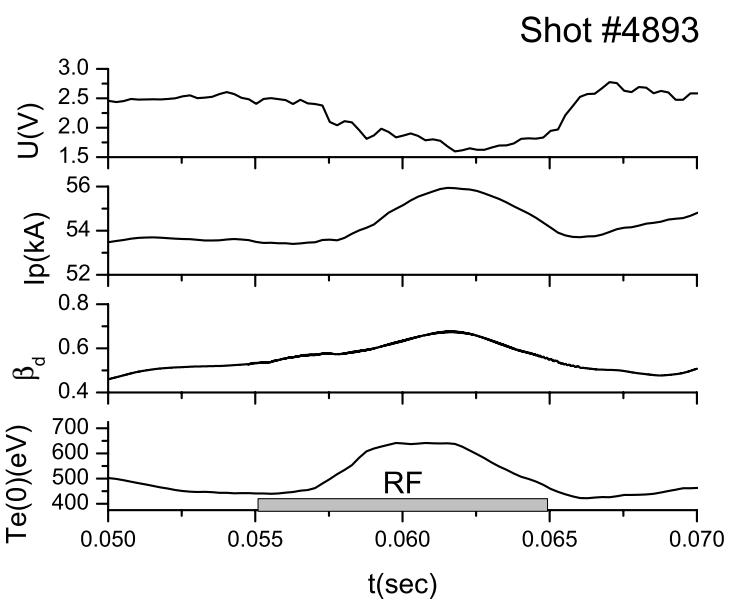

Figure 6. Details of the signals $U, I_{P}, \beta_{d}$ of plasma discharge \#4893.

As it was shown in [2], the character of the loop voltage evolution in a tokamak discharge during noninductive current drive depends on the radial position of the resonance zone, where the driven current is generated. The results of loop voltage reconstruction for different radial positions of the RF driven current $x=\frac{r}{a}$ in TCABR are shown in Fig.7. Here the origin of axis $t$ is moved to the plasma current start up time, i.e. to $t \approx 29 \mathrm{msec}$. It is seen that the waveform of the loop 
voltage response is quite different for $x=0.6$ to 0.8 (Fig.7a), $x=0.45$ to 0.65 (Fig.7b), $x=0.15$ to 0.45 (Fig.7c), in spite of the fact that the waveform of RF driven current was the same. This phenomenon can be used as diagnostic tool for finding the excited Alfvén wave spectrum, which is responsible for the observed current drive. This procedure has to include decomposition of the experimentally obtained voltage drop in a series of basic loop voltage responses, which are similar to ones shown in Fig.7.
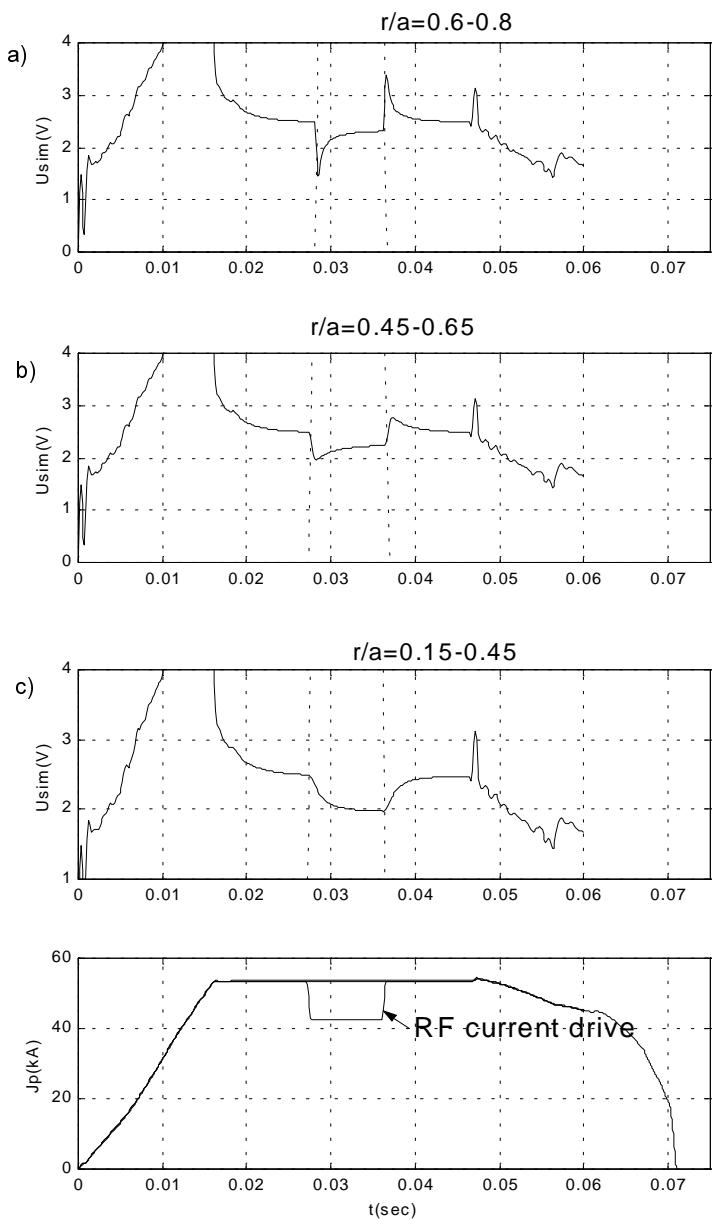

Figure 7. Numerical reconstruction of loop voltage response to noninductive current drive for different radial positions of RF power input zone.

In our case the shape of the experimentally measured loop voltage curve has been fitted to the shape of the reconstructed loop voltage curves, which were calculated for different radial positions of driven current. The initial data for numerical procedure were obtained from the experimental data, which are stored in the TCABR data base. This analysis shows that the main part of the RF driven current, which can have been generated in plasma discharge \#4893, flows in the inner plasma regions.

At the next step of our analysis, we suppose that there are not any RF driven current and that the to- tal magnitude of the loop voltage drop is due to plasma electron heating. The results of loop voltage reconstruction for this case are shown in Fig.8. It is seen that the reconstructed loop voltage curve, which is marked by circles, fits the experimental curve (solid line) rather well. The plasma conductivity was calculated by the Spitzer formula with $Z_{e f f}=4$. The electron temperature, which was used in fitting procedure, is shown at the bottom of Fig.8. It is seen that for reasonable explanation of the observed loop voltage drop we have to assume that the electron temperature increases due to the Alfvén wave heating, i.e., $\Delta T_{e} \simeq 180-200 \mathrm{eV}$. At the same time data of diamagnetic measurements ( $\beta$ in Fig.8) show smaller values of $\Delta T_{e}$. For elimination of this discrepancy, we have to relate part of loop voltage drop to noninductive current generation.
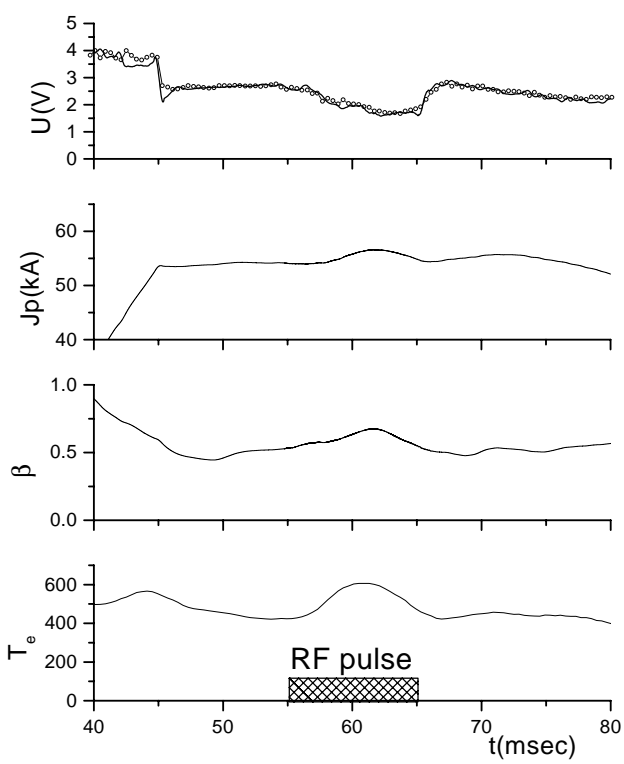

Figure 8. Loop voltage reconstruction for shot \#4893 without RF current drive.

Now we assume that some noniductive current is driven by Alfvén waves in inner plasma regions, and adjust the reconstructed loop voltage to this case. The result of the simulation is shown in Fig.9. It is seen from the plasma current curves that during $\mathrm{RF}$ pulse some part of ohmic current is substituted by RF driven current, which amounts to $I_{C D} \simeq 8 \mathrm{kA}$. The reconstructed loop voltage now fits the experimentally measured values with a smaller plasma temperature increase $\Delta T_{e} \leq 150 \mathrm{eV}$ and it better corresponds to the diamagnetic measurements. In this experiment the RF power that has been dissipated in the plasma amounted to a value $\widetilde{P}_{R F} \geq 80 \mathrm{~kW}$, and the efficiency of RF current drive can be estimated as $\eta=\frac{I_{C D}}{\widetilde{P}_{R F}} \leq 0.1-0.12$ $(\mathrm{A} / \mathrm{W})$. This efficiency is close to the values that were 
obtained during Alfvén wave current drive in PHAEDRUS - T tokamak, at smaller plasma densities [3]. Rather high efficiency of current drive in TCABR can be connected with generation of current in inner plasma regions, where the effect of trapped particles plays smaller role. Further experimental studies with more accurate diagnostic tools are necessary for verification of these results.
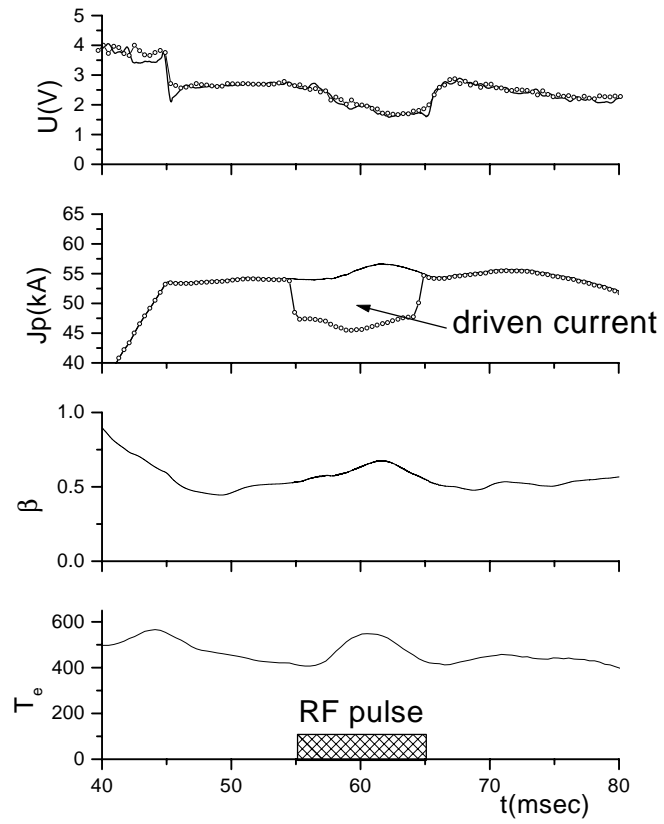

Figure 9. Loop voltage reconstruction for shot \#4893 with $\mathrm{RF}$ current drive included.

The calculated plasma current profile for these simulations is shown in Fig.10. It is worthwhile to note that some evidence of current profile modification can be found in the MHD activity modification, which was registered by Mirnov probes during Alfvén wave excitation.

\section{Conclusion}

In the course of Alfvén wave experiments in TCABR tokamak the regime of effective antenna- plasma coupling was found with RF power input $\widetilde{P}_{R F}>80 \mathrm{~kW}$.

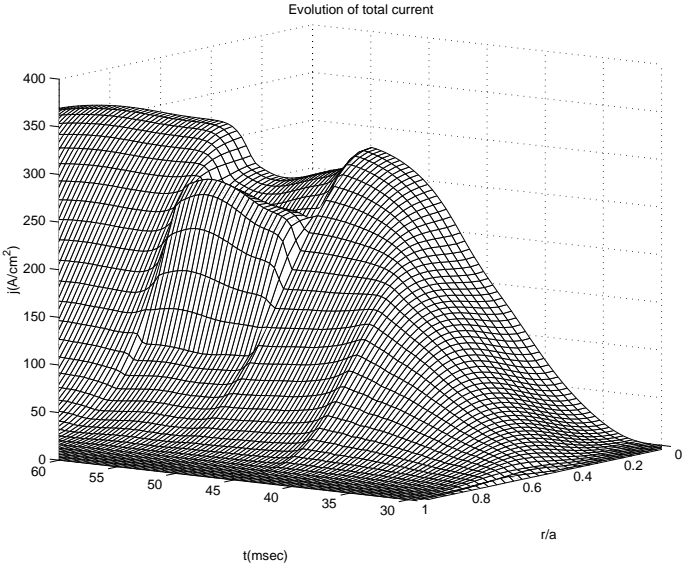

Figure 10. Current profile evolution in plasma shot \#4893 with Alfvén wave current drive.

The results of experiments on Alfvén wave current drive and plasma heating have been analyzed with the help of numerical code for simulation of toroidal electric field diffusion.

The numerical reconstruction of the loop voltage response to the RF power input suggests the possibility of RF current drive $I_{C D} \simeq 8 \mathrm{kA}$ with an efficiency $\eta \simeq 0.1$ $\mathrm{A} / \mathrm{W}$, however more accurate diagnostics are necessary.

\section{Acknowledgement}

This work has been supported by Conselho Nacional de Pesquisa e Desenvolvimento Científico e Tecnológico, Fundação de Amparo à Pesquisa do Estado de São Paulo e Financiadora de Estudos e Projetos, Brasil.

\section{References}

[1] Ya. I. Kolesnichenko, V.V. Parail, G.V. Pereverzev, Rev. Plasma Phys. edited by Kadomtsev B.B., Consultants Bureau, New York, 17, 3 ,1990.

[2] C. Litwin, Phys. Plasmas 2, 4542 (1995).

[3] C. Litwin, N. Hershkowitz, S. Wukitch, T. Intrator, M. Vucovic, D. Brouchous, R. Breun, and M. Harper, Phys. Plasmas 2, 4551 (1995).

[4] L.F. Ruchko, E. Ozono, R.M.O. Galvão, I.C. Nascimento, F.T. Degasperi, E. Lerche, Fusion Engineering and Design 43, 15 (1998).

[5] L.F. Ruchko, M.C. Andrade, R.M.O. Galvão. Nuclear Fusion, 36, 503 (1996). 